\title{
BMJ Open Assessment of adherence to oral nutritional supplementation and exploration of barriers and facilitators in patients after gastric cancer surgery: a mixed methods study protocol
}

\author{
Guang-ying Wan, ${ }^{1}$ Hui Xue, ${ }^{2}$ Hua Yuan, ${ }^{3}$ Xin Wang, ${ }^{4}$ Hui-qin Li, ${ }^{1}$ Xiuying Zhang (1) ${ }^{1}$
}

To cite: Wan G, Xue H, Yuan $\mathrm{H}$, et al. Assessment of adherence to oral nutritional supplementation and exploration of barriers and facilitators in patients after gastric cancer surgery: a mixed methods study protocol. BMJ Open 2021;11:e044308. doi:10.1136/ bmjopen-2020-044308

- Prepublication history and additional materials for this paper is available online. To view these files, please visit the journal online (http://dx.doi. org/10.1136/bmjopen-2020044308).

G-yW and $\mathrm{HX}$ contributed equally.

Received 31 August 2020 Revised 09 February 2021 Accepted 03 March 2021
D Check for updates

(C) Author(s) (or their employer(s)) 2021. Re-use permitted under CC BY-NC. No commercial re-use. See rights and permissions. Published by BMJ.

For numbered affiliations see end of article.

Correspondence to

Xiuying Zhang; z_xy@jlu.edu.cn

\section{ABSTRACT}

Introduction Postoperative malnutrition is a major issue in patients with gastric cancer. The European Society for Clinical Nutrition and Metabolism recommends oral nutritional supplements (ONS) as a first-line nutritional therapy to prevent malnutrition in patients with cancer. However, adherence to ONS is unsatisfactory. The overall aim of this study was to evaluate the adherence of patients with gastric cancer to ONS and to explore the promoting and hindering factors.

Methods and analysis In this study, we will use mixed methods with an explanatory sequential approach for data collection and analysis. In the first phase, a 12-week longitudinal study will be performed to identify changes in trends of oral nutritional supplementation adherence in 135 patients with gastric cancer, the impact of adherence on nutritional indicators and clinical outcomes and ONS adherence-related factors. The primary endpoints include patient adherence to ONS, weight, body mass index and grip strength followed by 30-day readmission rate, complications and adverse reactions. In the second stage, qualitative research will be implemented to provide in-depth insight into the quantitative results. Finally, quantitative and qualitative results will be combined for analysis and discussion to put forward suggestions for improving patients' ONS adherence.

Ethics and dissemination This research protocol has been approved by the Ethics Committee of the School of Nursing, Jilin University, China (No. 2019101601). Results will be disseminated in peer-reviewed journals and conferences, and sent to participating practices. Trial registration number ChiTR2000032425.

\section{INTRODUCTION}

As the fifth most common malignancy and the third most common cause of cancer-related deaths in the world, gastric cancer accounted for 841000 deaths in $2013 .{ }^{1}$ Radical gastrectomy remains the primary treatment for gastric cancer. ${ }^{2}$ However, patients' gastric reservoir function is depleted after surgery, ${ }^{3}$ leading to dramatic weight loss within the first 3 months post gastrectomy. ${ }^{4}$ Studies
Strengths and limitations of this study

- This protocol first details a complex and deeper understanding of oral nutritional supplement (ONS) adherence in patients after gastric cancer surgery using mixed methods with an explanatory sequential approach.

- Based on the definition of adherence, we will use two indicators, namely, dose and time, to measure patient adherence and will divide patient adherence into four situations to evaluate the effect of four adherence situations on nutritional outcome indicators.

- The qualitative phase will be performed to gain an in-depth understanding of the factors leading to changes in the adherence of patients with gastric cancer taking ONS over 12 weeks, it is very important for future strategies to improve patient adherence to ONS after gastric cancer surgery.

- Due to the restrictions of China's reimbursement policy, patients cannot be fully reimbursed for the ONS taken for 12 weeks, so when enrolling patients, selection bias may arise due to different economic conditions, thereby affecting patient adherence.

- The doctor will be prescribing ONS for 2 weeks, and all patients will purchase them by themselves until 12 weeks after the operation, which may lead to information bias and potentially leading to higher patient adherence in the first 2 weeks.

have shown that $19 \%-83 \%$ of patients suffer from malnutrition following gastrointestinal surgery, ${ }^{5-7}$ and their malnutrition status can take up to 1 year to recover. ${ }^{4}$

Malnutrition is associated with increased postoperative complications and morbidity, ${ }^{89}$ reduced quality of life ${ }^{6}$ and longer hospital stays. ${ }^{10}$ Furthermore, malnutrition 3 months after surgery was significantly associated with reduced overall survival (OS) and causespecific survival (CSS) in patients with gastric cancer. ${ }^{4}$ Therefore, it is of great significance 
to prevent malnutrition in patients with gastric cancer after surgery.

The European Society for Clinical Nutrition and Metabolism (ESPEN) recommends oral nutritional supplements (ONS) as a type of enteral nutrition to prevent malnutrition in patients with cancer. ${ }^{11}$ Studies have shown that ONS improves the nutritional status of patients with cancer after surgery. ${ }^{12} 13$ A previous study showed that taking ONS for 12 weeks prevented patients from losing $1.1 \mathrm{~kg}$ (2\% of body weight) after surgery. ${ }^{14}$ However, adherence to ONS is unsatisfactory, with rates as low as $42 \% .{ }^{15}$ Therefore, improving patient adherence to ONS is crucial to maintaining the nutritional status of patients with gastric cancer.

At present, studies on ONS adherence in postoperative gastric patients are limited. Adherence is often described in previous studies as a secondary outcome indicator, so these studies provided limited reference for the development of ONS strategy for postoperative gastric patients. Although ONS is a first-line nutritional therapy recommended by ESPEN, ${ }^{11}$ not all countries routinely prescribe ONS to prevent malnutrition in patients with cancer. ${ }^{16} 17$ Some studies have found that patients' illness perceptions affect medication adherence, ${ }^{18}{ }^{19}$ and patients' illness perceptions will change over time. ${ }^{20}$ Therefore, the research presented above leads to the assumption that the adherence of ONS will show a dynamic change in postoperative gastric patients. Given that patients may need to take ONS for a long time, it is important to explore the reasons for low patient adherence to gain a deeper understanding of the reasons patients decide to continue or stop taking ONS from their perspective. In this paper, we describe a mixed methods protocol for examining dynamic ONS adherence changes and exploring barriers and facilitators of ONS adherence in patients after gastric cancer surgery, which provides an explanatory sequential approach to bring in-depth insight into ONS adherence in patients after gastric cancer surgery. We present the following article in accordance with the SPIRIT (Standard Protocol Items: Recommendations for Interventional Trials) reporting checklist (available in online supplemental appendix 1).

\section{AIMS, OBJECTIVES AND RESEARCH QUESTIONS Study aims}

The overall aim of this study is to evaluate the adherence of patients with gastric cancer to ONS, explore the promoting and hindering factors, and provide a reference for the formulation of relevant strategies for ONS adherence in patients after gastric cancer surgery in the future.

\section{The main research questions}

- How will adherence change in patients taking ONS for 12 weeks after gastric cancer surgery?

- How will different situations of adherence affect nutritional indicators and clinical outcomes?
- What factors cause differences in the adherence of patients to ONS after gastric cancer surgery?

- How do patient experiences differ with respect to different adherence rates to ONS after gastric cancer surgery?

\section{The objectives of the study}

The first phase: objectives of the quantitative study

- Determine the levels and changes in gastric cancer patient adherence to ONS at different follow-up points.

- Determine the relationship between patient adherence and nutritional indicators, such as weight, body mass index (BMI), grip strength, 30-day readmissions, complications and adverse reactions.

- Determine the association between adherence to ONS and demographic characteristics of patients (age, sex, living arrangement and so on) and the texture of ONS.

The second phase: objectives of the qualitative study

- Further explore factors that promote and hinder gastric cancer patient adherence to ONS in the quantitative study.

- Explore factors not found in the quantitative study that may affect patient ONS adherence.

\section{METHOD AND ANALYSIS}

\section{Study design}

This study will use an explanatory sequential mixed method design to identify the change in trends of ONS adherence and explore barriers and facilitators to adherence in patients after gastric cancer surgery. The study design includes two phases ${ }^{21}$ (figure 1). In the first phase, we will conduct a 12-week longitudinal study to observe changes in the adherence of patients on ONS after gastric cancer surgery and to identify factors affecting differences in adherence. After statistical analysis of the quantitative data, we will design and perform the second qualitative phase of the study. The qualitative study will be informed by a purposive sample of patients from the quantitative study, the interview guide will be informed by the quantitative study, and in-depth insight will be provided into the quantitative results. Finally, quantitative and qualitative results will be combined for discussion. Quantitative research will begin in March 2021 and is expected to be completed in July 2021. The qualitative research will follow in August 2021.

\section{Patient and public involvement}

Neither patients nor the public were involved in the design, conduct, reporting or dissemination plans of this research.

\section{Stage 1: quantitative study}

We will conduct a 12-week longitudinal study (each week will serve as a follow-up point) to evaluate adherence to ONS in patients with gastric cancer after surgery. At the same time, we will also evaluate the relationship 


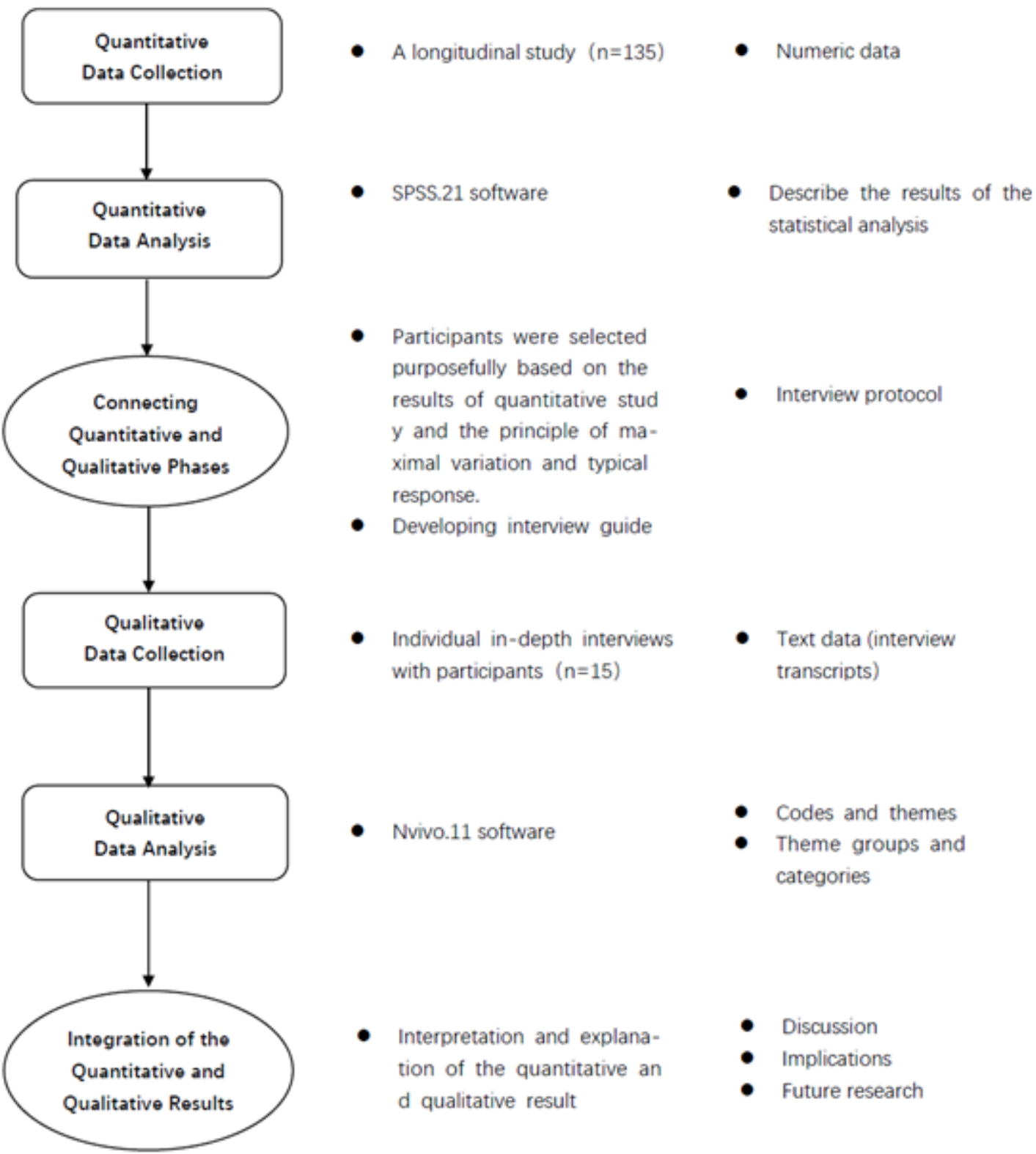

Figure 1 Study diagram.

between adherence and body weight, BMI, grip strength, 30-day readmissions, complications and adverse reactions (figure 2).

\section{Sample size and sampling method}

Sample size was calculated based on the Bunout et al study. ${ }^{22}$ Considering $\alpha=0.05$ and $\mathrm{Z}_{\alpha / 2}=1.96$, the sample size needed was estimated to be 113 . Assuming a $10 \%$ dropout rate and $10 \%$ mortality, the final sample size was determined to be 135 . The calculation formula is as follows: $\mathrm{n}=(\mathrm{Z} \alpha / 2 \sigma / \delta)^{2}$.

Before this study, we found that not all doctors routinely prescribed ONS to prevent malnutrition in patients with cancer in China. A physician order for ONS is a prerequisite for patient adherence to ONS, so we will use the purposive sampling method to select patients who already have an ONS prescription during the first 7 postoperative days in the gastrointestinal surgery department of a tertiary class hospital in Jilin Province. A member of the research team will explain the study to patients, and eligible patients will be invited to join the study and sign the informed consent form. Inclusion and exclusion criteria are shown in table 1.

\section{Data collection and schedule}

Baseline data collection

Collection of quantitative data will begin after obtaining informed consent. Demographic and socioeconomic data and clinical backgrounds will be collected through a general information questionnaire. Body weight (in $\mathrm{kg}$ ), 


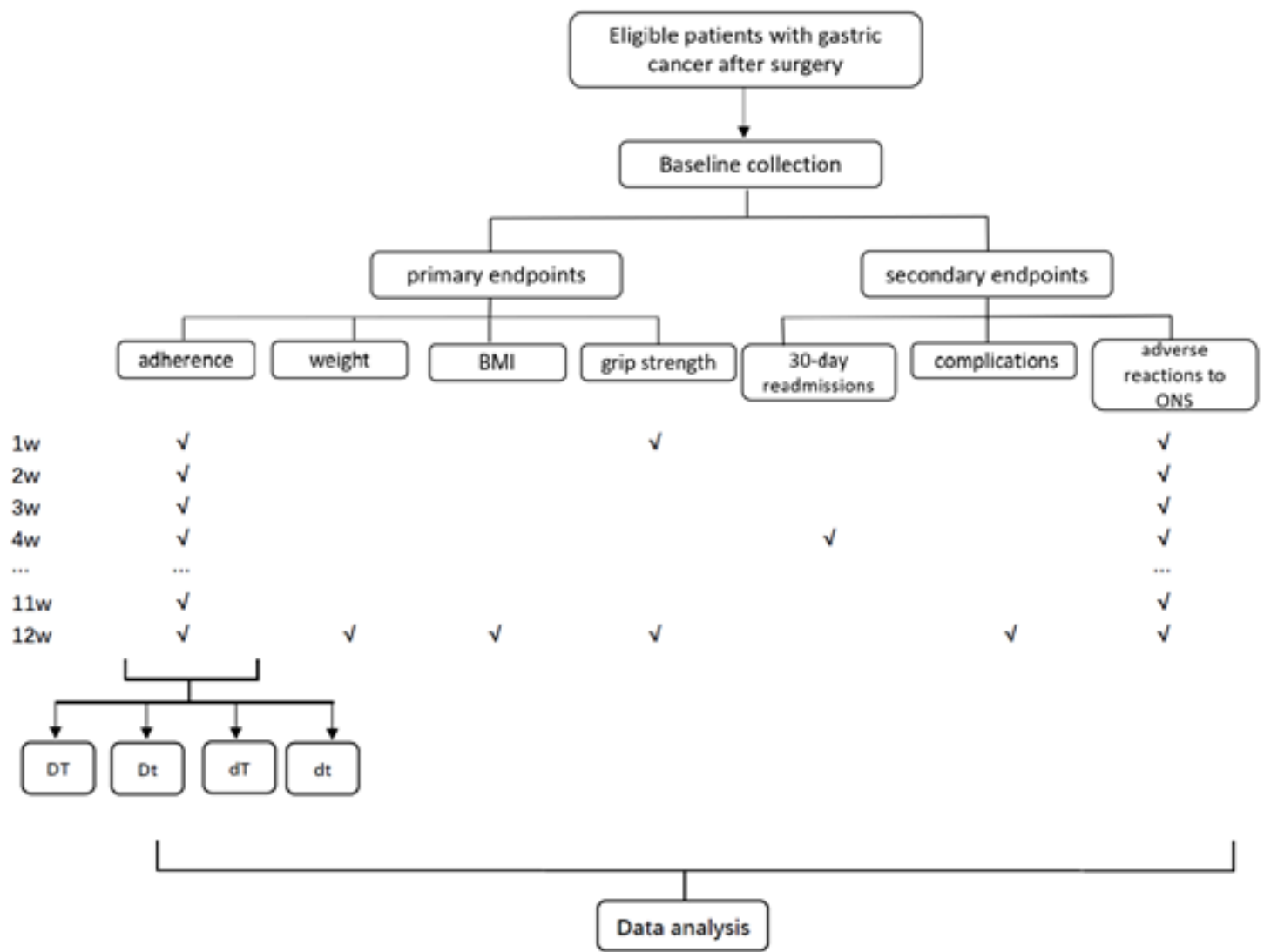

Figure 2 Quantitative study design flow chart. BMI, body mass index; dt, both the dosage and the number of days did not meet the doctor's requirements; DT, both the dosage and the number of days meet the doctor's requirements; dT, the daily dosage did not meet the doctor's order, and the days of taking reached the prescribed days; Dt, the daily dosage reached the prescribed amount, and the days of taking did not meet the doctor's requirements; ONS, oral nutritional supplements; W, week.

\begin{tabular}{ll}
\hline Table 1 Inclusion and exclusion criteria \\
\hline Inclusion & Exclusion \\
\hline $\begin{array}{l}\text { Patients with stage I, II } \\
\text { or III gastric cancer were } \\
\text { diagnosed by pathology } \\
\text { before surgery }\end{array}$ & $\begin{array}{l}\text { Diagnosis of double } \\
\text { cancer }\end{array}$ \\
Patients who had distal or & Allergy to milk, soy bean, \\
total gastrectomy & wheat or salmon \\
Age 18 years or order & $\begin{array}{l}\text { Patients with mental } \\
\text { illness, impaired }\end{array}$ \\
& $\begin{array}{l}\text { consciousness and } \\
\text { inability to communicate } \\
\end{array}$ \\
& normally
\end{tabular}

- PG-SGA Grades B or C

No gastrectomy, radiation, chemotherapy before surgery

- Patients have the ability to ingest food orally

Patients or his/her family members had a smartphone on which they could use WeChat*

- Patients written informed consent

*WeChat: as one of the popular social media apps in China.
BMI and grip strength will be assessed preoperatively to monitor patients' nutritional status.

\section{Primary endpoints}

The primary endpoints include patient adherence and nutritional status at 12 weeks post gastrectomy (weight, BMI, grip strength). Details of the schedule are described in table 2.

Adherence will be measured through the patient's 'weekly ONS diary'. In this study, ONS will be prescribed by the patients' attending physician, who will prescribe 400 kcal of ONS to the patient every day. Patients' attending physician will prescribe liquids or powders according to her/his usual practice. When ONS is prescribed, a researcher will record ONS-related factors, including name and texture. After discharge, participants will be required to keep a daily 'weekly ONS diary', including the amount of daily ONS consumed and adverse reactions. Each patient in the study will be enrolled in a group chat on WeChat (as one of the popular social media apps in China, WeChat is widely used for patient disease guidance and education ${ }^{23}$ ), and the researcher will ask patients for 'weekly ONS diary' photos by WeChat at each follow-up time point. Patient weight, BMI and grip strength will be remeasured 3 months after surgery when the patient is re-examined. 
Table 2 Content for the schedule of enrolment and assessments

Study period

\begin{tabular}{|c|c|c|c|c|c|c|c|c|c|c|c|c|c|}
\hline \multirow[b]{2}{*}{ Time point } & \multirow{2}{*}{$\frac{\text { Enrolment }}{\text { Day -7 0 }}$} & \multicolumn{11}{|c|}{ Post-allocation (12 weeks) } & \multirow{2}{*}{$\begin{array}{l}\text { Close-out } \\
3 \text { months }\end{array}$} \\
\hline & & 1 & 2 & 3 & 4 & 5 & 6 & 7 & 8 & 9 & 10 & 11 & \\
\hline \multicolumn{14}{|l|}{ Enrolment } \\
\hline Informed consent & $x$ & & & & & & & & & & & & \\
\hline Baseline data & $x$ & & & & & & & & & & & & \\
\hline
\end{tabular}

\begin{tabular}{|c|c|c|c|c|c|c|c|c|c|c|c|c|c|}
\hline \multicolumn{14}{|l|}{ Assessments } \\
\hline \multicolumn{14}{|l|}{ Primary endpoints } \\
\hline Adherence & & $x$ & $x$ & $x$ & $x$ & $x$ & $\times$ & $x$ & $x$ & $x$ & $x$ & $x$ & $x$ \\
\hline BMI & $x$ & & & & & & & & & & & & $x$ \\
\hline Grip strength & $x$ & & & & & & & & & & & & $x$ \\
\hline \multicolumn{14}{|l|}{ Secondary endpoints } \\
\hline 30-day readmissions & & & & & $x$ & & & & & & & & \\
\hline Complications & & & & & & & & & & & & & $x$ \\
\hline Adverse reactions & & $x$ & $x$ & $x$ & $x$ & $x$ & $x$ & $x$ & $x$ & $x$ & $x$ & $x$ & \\
\hline
\end{tabular}

BMI, bodymass index; ONS, oral nutritional supplements.

To rule out the effects of daily oral food intake on the study results, calories in the patient's daily diet will be calculated at each follow-up based on the local dietary assessment form (1-5 points) in China. ${ }^{24}$

\section{Secondary endpoints}

Secondary endpoints are as follows: (1) 30-day readmissions, where readmission will be defined as return to hospitalisation for any disease diagnosed within 30 days after surgery; (2) infective complications, which will include wound infection, respiratory tract infection and infective diarrhoea $^{1225}$; and (3) adverse reactions to ONS, which will include bloating, diarrhoea, nausea and vomiting. ${ }^{26}$ Details of the schedule are described in table 2.

To illustrate overall trends of ONS adherence in patients with gastric cancer after surgery over time, we will calculate the adherence by proportion of ONS consumed (the percentage of prescribed dosage consumed $)^{17}$ and create the ONS adherence curve based on the average ONS adherence at each time point. Adherence is defined as 'Voluntary cooperation of the patient in taking drugs or medicine as prescribed. This includes timing, dosage, and frequency. ${ }^{, 27}$ Adherence does not only encompass dose adherence but also includes duration of use, so we will use the second method to calculate adherence by the proportion of days covered (the percentage of prescribed days covered). ${ }^{28}$ To elucidate the status of ONS adherence in patients at different time points and provide heterogeneous samples representing different levels of adherence for subsequent qualitative studies, we will use both methods to describe adherence changes. The following four situations will occur at this point: (1) both the dose and the number of days meet the doctor's requirements (DT); (2) the daily dose reached the prescribed amount, but the days of taking ONS did not meet the doctor's requirements (Dt); (3) the daily dosage did not meet the doctor's order, but the days of taking ONS reached the prescribed days $(\mathrm{dT})$; and (4) neither the dosage nor the number of days met the doctor's requirements $(\mathrm{dt})$. Therefore, at each time point, patients will be divided into four groups according to their adherence. Next, we will use a bar graph to illustrate the proportion and changes among the four groups of people at different time points. Finally, we will compare differences in outcome indicators among the four groups (figure 2).

Measurement data will be described as the mean (SD), independent sample t-tests will be used to compare the average level of independent data, and paired sample t-tests will be used to compare the average level of paired data. One-way analysis of variance will be used to compare data in different groups, and pairwise comparisons will be performed with the Least-Significant Difference method. Categorical variables will be expressed as percentages, and the $\chi^{2}$ test will be used for comparisons between groups.

\section{Stage 2: qualitative study}

In this stage, we will conduct qualitative research to explain the results of the quantitative phase and further explore barriers and facilitators of adherence to ONS from the perspective of patients with gastric cancer (figure 3 ). 


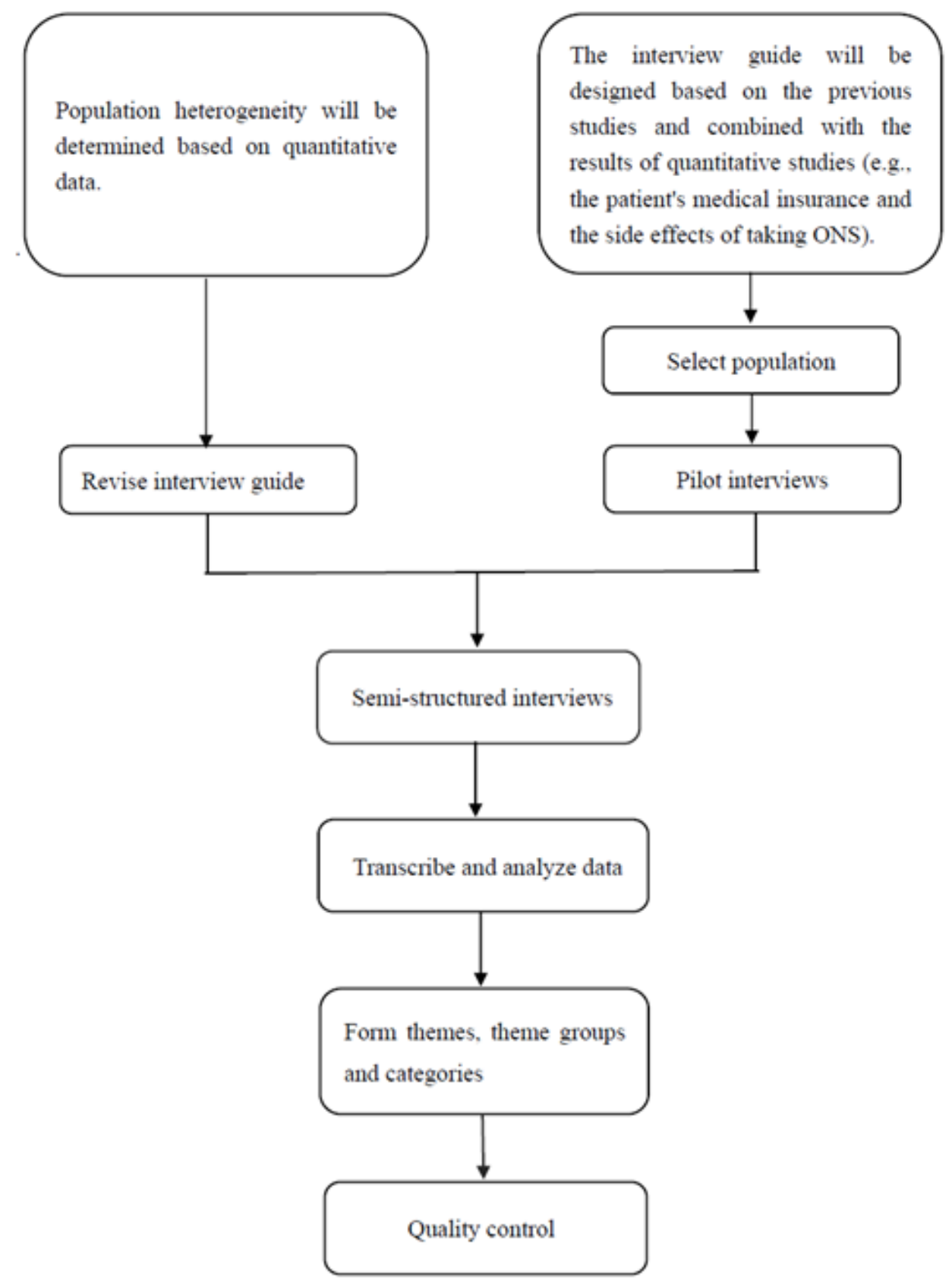

Figure 3 Qualitative study design flow chart. ONS, oral nutritional supplements.

\section{Sampling method}

We will use purposive sampling to select participants. To ensure the sampling follows the principles of typical response and maximal variation, we will select participants who represent the results of the quantitative phase (they differ in age, education, income, adherence and other aspects) to better explain the quantitative research with qualitative research. Recruitment of patients will be discontinued when the qualitative data become saturated. ${ }^{29}$

\section{Designing the interview guide}

In this explanatory sequential mixed methods study, designing the interview guide will be an important link between the qualitative and quantitative research. The quantitative results will not only guide the sampling procedure but also point towards questions in the qualitative phase. We will design interview guides for qualitative research based on the results of quantitative research. The qualitative data will provide more depth and insight into the quantitative results. In addition, we will give the formed interview guide to a psychologist for review so that the interview can proceed more smoothly and obtain more effective information. Pilot interviews will be conducted with a small number of participants, and the interview guide will be further revised according to the information obtained from the pilot interviews. By doing 
this, we will ensure that the interview guide will be culturally sensitive and easier for patients to understand.

\section{Data collection}

Data will be collected using open-ended questions through in-depth semistructured interviews. The interview will begin with the question 'How well are you recovering?' To understand patient perceptions of ONS, we will ask, 'Do you feel that ONS has any effect on your recovery from the disease?' Then, based on the results of the first phase of the study, we will ask patients who have different types of adherence questions, such as 'Can you tell me why you stopped taking ONS?' Finally, we will encourage patients to make suggestions to improve adherence to ONS. For example, 'Do you have any suggestions?' and 'Anything else you'd like to add that we haven't covered?'

In the interview, we will use 'Why?', 'What do you mean?', and 'Can you explain this?' to further understand patients' experiences. To ensure that effective information is obtained from the interview, all patients will be interviewed in a comfortable setting. Following consent, interviews will be recorded and transcribed verbatim. During the interview, we will record non-verbal data from patients in a notebook, such as intonation, movements, gestures, expressions and so on.

\section{Data analysis}

In our study, data collection and analysis will be conducted simultaneously. After each interview, researchers will transcribe the recording into words in a timely manner and mark the emotional responses of interviewees in the corresponding position of the text. To preserve the original style of the data, we will use the patient's dialect whenever possible. We will use NVivo and Colaizzi's sevenstep analysis methods to manage and analyse qualitative data, respectively. Researchers will carefully and repeatedly read all interview materials and extract relevant statements and meanings. Then, researchers will search for common concepts or characteristics of meanings and form themes, theme groups and categories.

\section{Validity and reliability/rigor}

'Rich rigour,' 'sincerity,' 'credibility' and 'resonance' are elements suggested by Tracy as denoting excellence in qualitative research. ${ }^{30}$ To improve and verify the accuracy of the results, we will give the transcript of the interview to the interviewees so that the patient can confirm whether the content is comprehensive. If there is any other content they want to add, they can add to the data directly. The corresponding author will read the content of the interview repeatedly to determine whether the results resonate with similar people, and the extracted text that contains themes will be reviewed by all the authors and thoroughly discussed. To ensure the codes and topics are professional and accurate, we will submit the generated codes and topics to psychologists for review. In addition, we will provide examples of the initial codes to other researchers who are not involved in this study to determine whether they have similar perceptions based on the text.

\section{DISCUSSION}

A previous study reported that the 12-month mortality rate is $26 \%$ among malnourished patients with gastric cancer. ${ }^{4}$ ONS is the preferred method of nutritional therapy, and some studies have shown that the effect of ONS is dosedependent, ${ }^{31-33}$ so maintaining high adherence to ONS plays an important role in the postoperative recovery of patients with gastric cancer. At present, adherence to ONS is generally low. Therefore, we need to identify the relevant factors affecting adherence to ONS in patients with gastric cancer. This is the first study to explore adherence to ONS in patients after gastric cancer surgery and will be performed via a mixed methods approach.

Malnutrition often leads to muscle dysfunction, especially thoracic muscle dysfunction, which often leads to pneumonia. ${ }^{34}$ Patients with malnutrition often develop impaired intestinal immune function that further aggravates nutrient digestion and absorption dysfunction. ${ }^{3536} \mathrm{In}$ addition, patient wound healing is also markedly affected by the state of malnutrition. ${ }^{37}$ Therefore, in our study, in addition to using body weight, BMI and grip strength as indicators to assess patients' nutritional status, we will also use complications, including wound infection, respiratory tract infection, and infective diarrhoea, and 30-day readmission rate as indicators to assess patients' postoperative recovery.

In other similar clinical studies, patient self-reporting was used to measure adherence, similar to using a patient's 'daily food diary' to record their daily intake (diet+ONS). ${ }^{38}$ We think that in our study, since the elderly ( $\geq 65$ years) make up a large portion of the participants, it would be difficult for patients to keep track of their diet in detail. ${ }^{17}$ Therefore, we will use an 'ONS diary' instead of a 'daily food diary' to record the dose of ONS consumed, and at each follow-up time, we will estimate patients' energy intake according to the 'Chinese diet assessment tool'. According to the assessment, the range corresponding to the score of this tool is $90 \%$, in accordance with the results of the standard diet survey. ${ }^{24}$ In addition, this scale has been used in other studies as a nutritional assessment tool. ${ }^{39} 40$

Due to China's medical policy, each doctor can only prescribe an approximately 2 week dose of ONS to patients, and patients will buy ONS at the community pharmacy when all their prescribed ONS has been used up. At present, in the Chinese market, the texture of ONS is primarily powder and liquid, but to observe the impact of different texture types of ONS (liquid or powder) on patient adherence, we will keep the original style of doctors prescribing ONS in our study. However, regardless of whether powder or liquid, we will use intact protein-based ONS.

To explore factors that facilitate and present barriers to patient adherence to ONS, we will use a mixed methods 
approach. This approach not only has the advantages of quantitative and qualitative research but also overcomes the limitations of the separate study approach. Recently, there was a quantitative study on ONS adherence in patients after gastrointestinal cancer surgery, ${ }^{26}$ but it only identified barriers to adherence of ONS based on reviewing previous studies to extract frequent reasons, without an in-depth understanding of patients' feelings, which may have limited the results. In other studies, changes in patient adherence to ONS at different times after surgery were not compared, which may lead to new findings in our study. Therefore, this study will have a more comprehensive and in-depth understanding of the facilitators and barriers of patient ONS adherence. To our knowledge, this method has not been previously used to specifically understand the influencing factors of ONS adherence in this group of patients. The key output of this study will be practice recommendations in relation to the development of an ONS strategy for postoperative gastric patients.

\section{ETHICS AND DISSEMINATION}

This research protocol has been approved by the Ethics Committee of the School of Nursing, Jilin University, China (No. 2019101601). Results will be disseminated in peer-reviewed journals and conferences, and sent to participating practices.

\section{Author affiliations}

${ }^{1}$ Department of Fundamental Nursing, School of Nursing, Jilin University, Changchun, China

${ }^{2}$ Department of Histology \& Embryology, College of Basic Medical Sciences, Jilin University, Changchun, China

${ }^{3}$ Department of Surgical Nursing, School of Nursing, Jilin University, Changchun, China

${ }^{4}$ Institution of Sports Medicine of Third Hospital, Peking University Third Hospital, Beijing, China

Contributors Conception and design: G-YW, HX, X-YZ. Administrative support: HX. Provision of study materials or patients: $\mathrm{HX}, \mathrm{HY}, \mathrm{XW}$. Collection and assembly of data: G-YW, HX, H-QL, X-YZ. Data analysis and interpretation: G-YW, HX. Manuscript writing: All authors. Final approval of manuscript: All authors.

Funding This work was supported by the National Natural Science Foundation of China (grant number 31800895); Higher Education Reform Project of Jilin Province (grant numbers JGJX2019D10, JGJX2019D6); Reform of Undergraduate Teaching Project in Jilin University (grant number 2019XYB252, 2019XYB295).

Competing interests None declared.

Patient consent for publication Not required.

Provenance and peer review Not commissioned; externally peer reviewed.

Supplemental material This content has been supplied by the author(s). It has not been vetted by BMJ Publishing Group Limited (BMJ) and may not have been peer-reviewed. Any opinions or recommendations discussed are solely those of the author(s) and are not endorsed by BMJ. BMJ disclaims all liability and responsibility arising from any reliance placed on the content. Where the content includes any translated material, BMJ does not warrant the accuracy and reliability of the translations (including but not limited to local regulations, clinical guidelines, terminology, drug names and drug dosages), and is not responsible for any error and/or omissions arising from translation and adaptation or otherwise.

Open access This is an open access article distributed in accordance with the Creative Commons Attribution Non Commercial (CC BY-NC 4.0) license, which permits others to distribute, remix, adapt, build upon this work non-commercially, and license their derivative works on different terms, provided the original work is properly cited, appropriate credit is given, any changes made indicated, and the use is non-commercial. See: http://creativecommons.org/licenses/by-nc/4.0/.

ORCID iD

Xiuying Zhang http://orcid.org/0000-0001-6006-2914

\section{REFERENCES}

1 Fitzmaurice C, Dicker D, et al, Global Burden of Disease Cancer Collaboration; Christina Fitzmaurice. The global burden of cancer 2013. JAMA Oncol 2015;1:505-27.

2 Japanese Gastric Cancer Association. Japanese gastric cancer treatment guidelines 2014 (VER. 4). Gastric Cancer 2017;20:1-19.

3 Bae JM, Park JW, Yang HK, et al. Nutritional status of gastric cancer patients after total gastrectomy. World J Surg 1998;22:254-61.

4 Fujiya K, Kawamura T, Omae K, et al. Impact of malnutrition after gastrectomy for gastric cancer on long-term survival. Ann Surg Oncol 2018;25:974-83.

5 Fukuda Y, Yamamoto K, Hirao M, et al. Prevalence of malnutrition among gastric cancer patients undergoing gastrectomy and optimal preoperative nutritional support for preventing surgical site infections. Ann Surg Oncol 2015;22:778-85.

6 Guo ZQ, Yu JM, Li W, et al. Survey and analysis of the nutritional status in hospitalized patients with malignant gastric tumors and its influence on the quality of life. Support Care Cancer 2020;28:373-80.

7 Ryu SW, Kim IH. Comparison of different nutritional assessments in detecting malnutrition among gastric cancer patients. World $\mathrm{J}$ Gastroenterol 2010;16:3310-7.

8 Choi WJ, Kim J. Nutritional care of gastric cancer patients with clinical outcomes and complications: a review. Clin Nutr Res 2016;5:65-78.

9 Yu W, Seo BY, Chung HY. Postoperative body-weight loss and survival after curative resection for gastric cancer. $\mathrm{Br} J$ Surg 2002;89:467-70.

10 Guner A, Kim SY, Yu JE, et al. Parameters for predicting surgical outcomes for gastric cancer patients: simple is better than complex. Ann Surg Oncol 2018;25:3239-47.

11 Arends J, Bachmann P, Baracos V, et al. ESPEN guidelines on nutrition in cancer patients. Clin Nutr 2017;36:11-48.

12 Beattie AH, Prach AT, Baxter JP, et al. A randomised controlled trial evaluating the use of enteral nutritional supplements postoperatively in malnourished surgical patients. Gut 2000;46:813-8.

13 Smedley F, Bowling T, James M, et al. Randomized clinical trial of the effects of preoperative and postoperative oral nutritional supplements on clinical course and cost of care. Br J Surg 2004;91:983-90.

14 Hatao F, Chen K-Y, Wu J-M, et al. Randomized controlled clinical trial assessing the effects of oral nutritional supplements in postoperative gastric cancer patients. Langenbecks Arch Surg 2017;402:203-11.

15 Grass F, Bertrand PC, Schäfer M, et al. Compliance with preoperative oral nutritional supplements in patients at nutritional risk--only a question of will? Eur J Clin Nutr 2015;69:525-9.

16 Brindisi M-C, Noacco A, Boudaoud Hansal AA, et al. Delivery of oral nutrition supplement in hospital: evaluation of professional practices in evaluation of nutritional status and representations of ONS by the caregivers and patients. Clin Nutr ESPEN 2020;35:85-9.

17 Seguy D, Hubert $H$, Robert J, et al. Compliance to oral nutritional supplementation decreases the risk of hospitalisation in malnourished older adults without extra health care cost: prospective observational cohort study. Clin Nutr 2020;39:1900-7.

18 Fennessy MM, Devon HA, Ryan C, et al. Changing illness perceptions and adherence to dual antiplatelet therapy in patients with stable coronary disease. J Cardiovasc Nurs 2013;28:573-83.

19 Kucukarslan SN. A review of published studies of patients' illness perceptions and medication adherence: lessons learned and future directions. Res Social Adm Pharm 2012;8:371-82.

20 Kaptein AA, Yamaoka K, Snoei L, et al. Illness perceptions and quality of life in Japanese and Dutch women with breast cancer. $J$ Psychosoc Oncol 2013;31:83-102.

21 Creswell J. Research design: qualitative, quantitative and mixed methods and approaches, 2003.

22 Bunout B, Barrera G, de la Maza P. Effects of nutritional supplementation and resistance training on muscle strength in free living elders. Results of one year follow. J Nutr Health Aging 2004;8:68-75.

23 Yu Q, Xu L, Li L, et al. Internet and WeChat used by patients with Crohn's disease in China: a multi-center questionnaire survey. BMC Gastroenterol 2019;19:97.

24 Cong MH, Shi HP. The invention of a simple diet self-assessment tool for cancer patients. Electron J Metab Nutr Cancer 2018;5:3. 
25 Sultan J, Griffin SM, Di Franco F, et al. Randomized clinical trial of omega-3 fatty acid-supplemented enteral nutrition versus standard enteral nutrition in patients undergoing oesophagogastric cancer surgery. Br J Surg 2012;99:346-55.

26 Lidoriki I, Schizas D, Mylonas KS, et al. Oral nutritional supplementation following upper gastrointestinal cancer surgery: a prospective analysis exploring potential barriers to compliance. J Am Coll Nutr 2020;39:650-6.

27 NLM mesh Homepage. Available: https://www.ncbi.nlm.nih.gov/ mesh

28 Stanton-Robinson C, Al-Jumaili AA, Jackson A. Evaluation of community pharmacist-provided telephone interventions to improve adherence to hypertension and diabetes medications. J Am Pharm Assoc 2003;2018:S120-4.

29 Francis $\mathrm{JJ}$, Johnston M, Robertson C, et al. What is an adequate sample size? Operationalising data saturation for theory-based interview studies. Psychol Health 2010;25:1229-45.

30 Tracy SJ. Qualitative quality: eight "big-tent" criteria for excellent qualitative research. Qualit Inquiry 2010;16:837-51.

31 de van der Schueren MAE, Laviano A, Blanchard H, et al. Systematic review and meta-analysis of the evidence for oral nutritional intervention on nutritional and clinical outcomes during chemo(radio) therapy: current evidence and guidance for design of future trials. Ann Oncol 2018;29:1141-53.

32 Dias Rodrigues V, Barroso de Pinho N, Abdelhay E, et al. Nutrition and immune-modulatory intervention in surgical patients with gastric cancer. Nutr Clin Pract 2017;32:122-9.
33 Kobayashi D, Ishigure K, Mochizuki Y, et al. Multi-institutional prospective feasibility study to explore tolerability and efficacy of oral nutritional supplements for patients with gastric cancer undergoing gastrectomy (CCOG1301). Gastric Cancer 2017;20:718-27.

34 Efthimiou J, Fleming J, Gomes C, et al. The effect of supplementary oral nutrition in poorly nourished patients with chronic obstructive pulmonary disease. Am Rev Respir Dis 1988;137:1075-82.

35 Chandra RK, Kumari S. Effects of nutrition on the immune system. Nutrition 1994;10:207-10.

36 van der Hulst RR, von Meyenfeldt MF, van Kreel BK, et al. Gut permeability, intestinal morphology, and nutritional depletion. Nutrition 1998;14:1-6.

37 Haydock DA, Hill GL. Impaired wound healing in surgical patients with varying degrees of malnutrition. JPEN J Parenter Enteral Nutr 1986;10:550-4.

38 Hubbard GP, Elia M, Holdoway A, et al. A systematic review of compliance to oral nutritional supplements. Clin Nutr 2012;31:293-312.

39 Shi HP, Chen W, Yang LQ. Fighting malnutrition in hospital: an introduction to hunger-free Hospital programme. Electron J Metab Nutr Cancer 2018:5:6.

40 Tian BW, Zhang JT, Zhang GY. Observation on the effect of grading and filing of nutrition intervention in patients with digestive tract cancer. Journal of Nursing 2019;26:4. 